\title{
INFLUÊNCIA DO TRATAMENTO ÁCIDO EM CASCA DE PINHÃO SOBRE OS PARÂMETROS DE EQUILÍBRIO E CINÉTICOS NO PROCESSO DE ADSORÇÃO DE CORANTE EM SOLUÇÃO AQUOSA
}

\author{
T. M. NEUBAUER, K. KELLER, B. O. RODRIGUES, E. L. FOLETTO, M. A. MAZUTTI e \\ A. CANCELIER
}

\begin{abstract}
Universidade Federal de Santa Maria, Departamento de Engenharia Química
E-mail para contato: thiagoneubauereq@gmail.com
\end{abstract}

\begin{abstract}
RESUMO - Este trabalho trata do estudo da adsorção do corante azul de metileno em solução aquosa por casca de pinhão, tanto in natura quanto tratada com $\mathrm{HCl}$ $1 \mathrm{M}$. As cascas de pinhão foram secas em estufa, trituradas e, por peneiramento, utilizou-se apenas partículas contidas entre 0,5 e $0,841 \mathrm{~mm}$. Os ensaios foram conduzidos em batelada, controlando-se a agitação e temperatura. Analisou-se a influência da temperatura, $\mathrm{pH}$ e concentração inicial de corante. Os parâmetros de adsorção foram obtidos a partir das isotermas de Langmuir e Freundlich, utilizando-se regressão linear e não linear. Os resultados mostraram melhor adaptação da cinética de pseudossegunda ordem e da isoterma de Langmuir aos dados experimentais, tanto para o pinhão in natura quanto para o tratado com $\mathrm{HCl}$. Determinou-se, a partir dos resultados de equilíbrio, que o pinhão tratado com $\mathrm{HCl}$ possui maior capacidade de adsorção e maior constante cinética.
\end{abstract}

\section{INTRODUÇÃO}

Atualmente, a má disposição final de esgotos e efluentes tem promovido um grande problema de agressão aos recursos hídricos e, se estes não forem tratados e dispostos devidamente, causam uma série de problemas físicos, químicos e biológicos num corpo d'água (Reife e Fremann, 1996).

Dentre os efluentes industriais, os corantes dispostos em soluções aquosas ainda constituem um sério problema, pois, mesmo em pequenas quantidades, conferem cor intensa e apresentam elevadas demandas química e bioquímica de oxigênio, constituindo um dos efluentes mais complexos problemáticos a serem tratados (AKSU, 2005).

As técnicas mais utilizadas para descoloração envolvem processos físico-químicos, principalmente mecanismos de adsorção, coagulação e precipitação, ultrafiltração, processos biológicos, além de processos oxidativos avançados (Papic et al., 2000; Karadag et al., 2006; Mohorcic et al., 2006; Bielska and Prochaska, 2007; Foletto et al., 2010). Alguns desses métodos geralmente são combinados, de forma a aumentar a eficiência do processo.

A adsorção é um dos métodos mais eficientes empregados na remoção de cor, odor, óleos e poluentes orgânicos. O carvão ativado é um dos adsorventes mais utilizados, no 
entanto, seu emprego é limitado em função do elevado custo. Por esse motivo, diversos estudos tem evidenciado a eficácia de adsorventes alternativos e de baixo custo (Rafatullah et al., 2010).

Neste contexto, este estudo avaliou a cinética e capacidade de adsorção do corante azul de metileno por casca de pinhão. O pinhão é a semente da araucária (Araucaria angustifólia), que é uma árvore que ocupa largas áreas no sul e no sudeste do Brasil e no leste da Argentina. A casca do pinhão é bastante fibrosa, própria para a adsorção, sendo normalmente descartada. Estima-se que cerca de 10 t/ano desta casca são produzidas e descartadas no Brasil (Lima et al, 2007).

Foram determinados e analisados os parâmetros de equilíbrio e da cinética de adsorção para a casca de pinhão in natura e tratada com ácido clorídrico. Analisou-se a influência da temperatura, $\mathrm{pH}$ e concentração inicial de corante. Os modelos de Langmuir e Freundlich foram utilizados para representar os resultados de equilíbrio de adsorção e os modelos de pseudoprimeira e pseudossegunda ordem foram avaliados para representar o comportamento da cinética de adsorção. Além disso, também foi feita uma comparação entre as metodologias de ajuste para os parâmetros, sendo avaliados os métodos de regressão linear e não linear.

\section{METODOLOGIA}

\subsection{Materiais}

A casca de pinhão foi obtida da semente adquirida comercialmente. A casca passou por um processo de moagem seguida de peneiramento, de maneira a se obter um material uniforme, com diâmetro entre 0,5 e 0,841 mm (20/35 mesh). Em seguida, o material foi lavado, secado em estufa a temperatura de $105^{\circ} \mathrm{C}$ por 24 horas e então armazenado em sacos plásticos. O tratamento ácido foi realizado em becker de $500 \mathrm{~mL}$, onde a casca de pinhão ficou em contato com $\mathrm{HCl} 1 \mathrm{M}$, por 24 horas, sob agitação magnética. A temperatura utilizada no tratamento foi de $25{ }^{\circ} \mathrm{C}$. Após o tratamento, o material foi lavado e seco em estufa. $\mathrm{O}$ adsorvente foi caracterizado por espectrometria de infravermelho (PerkinElmer FT-IR), por meio da qual se avaliou a influência do tratamento ácido sobre a superfície do sólido.

O corante utilizado como modelo nos ensaios de adsorção foi o azul de metileno (Sigma-Aldrich). Os demais reagentes utilizados foram o ácido clorídrico e hidróxido de sódio, ambos obtidos comercialmente da Sigma-Aldrich.

\subsection{Experimentos de Adsorção em Batelada}

Os experimentos envolvendo o processo de adsorção do corante azul de metileno pela casca do pinhão, tanto in natura quanto tratado com $\mathrm{HCl}$, foram realizados em batelada, em banho dubnoff com controle de temperatura e agitação. Em todos os ensaios a agitação foi mantida constante em $100 \mathrm{rpm}$. Foram utilizados erlenmeyers de $250 \mathrm{~mL}$, onde adicionava-se uma massa conhecida de adsorvente em $100 \mathrm{~mL}$ de solução aquosa do corante. Inicialmente, os ensaios avaliaram a influência do $\mathrm{pH}$ (faixa de 2,5 a 12), o qual foi ajustado pela adição de $\mathrm{HCl} 0,1 \mathrm{M}$ ou $\mathrm{NaOH} 0,1 \mathrm{M}$. Também foram avaliadas a temperatura (faixa de 20 a $45^{\circ} \mathrm{C}$ ) e a concentração de corante (faixa de $60 \mathrm{mg} . \mathrm{L}^{-1}$ a $2000 \mathrm{mg} . \mathrm{L}^{-1}$ ). No decorrer dos experimentos uma alíquota de $3 \mathrm{~mL}$ de solução era coletada e centrifugada para determinação da 
concentração de corante. Todas as amostras foram analisadas em espectrofotômetro UVVisível Shimadzu $1650 \mathrm{C}$, em $\lambda_{\max }=665 \mathrm{~nm}$.

A capacidade de adsorção do corante foi calculada de acordo com a Equação 1.

$$
q_{e}=\frac{\left(C_{0}-C_{e q}\right) V}{W}
$$

onde $q_{e}\left(\mathrm{mg} \cdot \mathrm{g}^{-1}\right)$ é a quantidade de corante adsorvido no equilíbrio, $C_{0}\left(\mathrm{mg} \cdot \mathrm{L}^{-1}\right)$ é a concentração inicial de corante, $C_{e q}\left(\mathrm{mg} \mathrm{L}^{-1}\right)$ é a concentração de corante na fase aquosa no equilíbrio, $W(\mathrm{~g})$ é a massa de adsorvente na solução e $V(\mathrm{~L})$ é o volume da solução.

\section{RESULTADOS E DISCUSSÃO}

Inicialmente, na Figura 1, apresentam-se os resultados quanto à caracterização do adsorvente (casca de pinhão in natura e tratada com $\mathrm{HCl}$ ) por espectrofotometria de infravermelho. A análise dos resultados permite concluir que o $\mathrm{HCl}$ reagiu com grupos funcionais da superfície da casca gerando grupos com maior caráter nucleofílico, por exemplo, a reação entre um aromático com $\mathrm{H}$ livre $\left(865,05 \mathrm{~cm}^{-1}\right)$ gerando um grupo fenol $\left(1161,56 \mathrm{~cm}^{-1}\right)$.

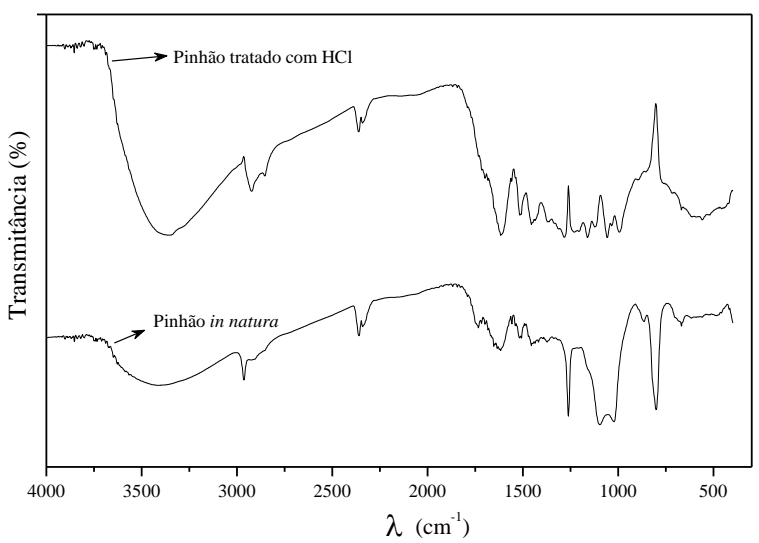

Figura 1 - Espectros de infravermelho para as amostras de casca de pinhão.

A Figura 2 apresenta o efeito do $\mathrm{pH}$ sobre a capacidade de adsorção para a casca de pinhão in natura e tratada com ácido clorídrico. Os resultados permitem verificar que à medida que a alcalinidade da solução aumenta também ocorre um aumento significativo da capacidade de adsorção do corante pela casca de pinhão, tanto in natura tanto para a tratada com ácido clorídrico. Esse comportamento já era esperado pelo fato do corante (azul de metileno) ter grupamentos com caráter básico, o que favorece a repulsão do meio mais alcalino com relação aos grupos nitrogenados que conferem alcalinidade ao corante. Para a casca de pinhão in natura observa-se que acima de $\mathrm{pH} 8$ já se tem um rendimento próximo ao máximo obtido, que foi de $72 \mathrm{mg} \cdot \mathrm{g}^{-1}$. Também observa-se que o maior aumento da capacidade de adsorção para o pinhão tratado com $\mathrm{HCl}$ ocorre apenas em $\mathrm{pH}$ bastante elevado. Este comportamento deve-se ao fato de que o pinhão tratado possui mais grupamentos aniônicos, conforme Figura 1 (grupo fenol em 1161,56 cm $\mathrm{cm}^{-1}$ e álcool secundário 
$1123,12 \mathrm{~cm}^{-1}$ ) os quais possuem maior afinidade com o íon hidrônio o que causa um impedimento repulsivo pelo fato do corante ter um grupamento catiônico.

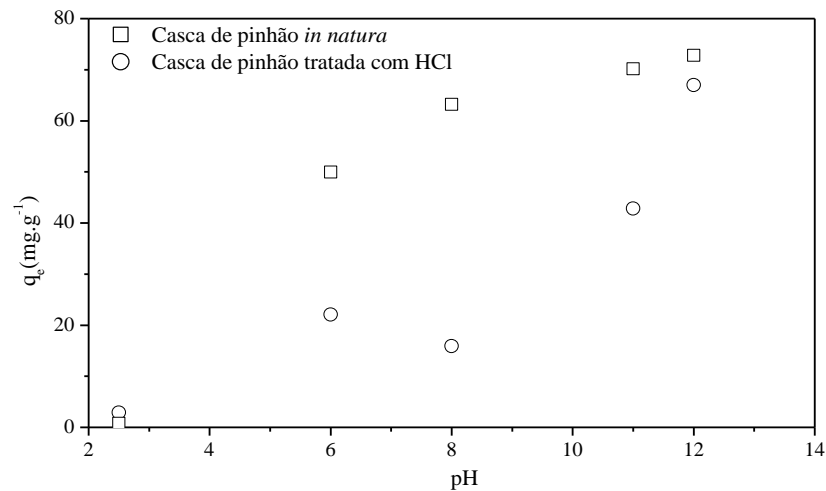

Figura 2 - Influência do pH na adsorção de azul de metileno por casca de pinhão.

Condições operacionais: $\mathrm{m}_{\text {adsorvente }}=0,015 \mathrm{~g}, \mathrm{C}_{0}=30 \mathrm{mg} \cdot \mathrm{L}^{-1}, \mathrm{~V}=30 \mathrm{~mL}, \mathrm{~T}=298 \mathrm{~K}$.

As Figuras 3 e 4 apresentam os resultados experimentais e isoterma de adsorção de Langmuir (Equação 2) obtida para o pH=12 e temperatura de $298 \mathrm{~K}$, para a casca de pinhão in natura e tratada com ácido clorídrico, respectivamente. Os parâmetros da isoterma de Langmuir (Tabela 1) foram determinados por regressão linear (Mínimos Quadrados) e regressão não linear (método Simplex).

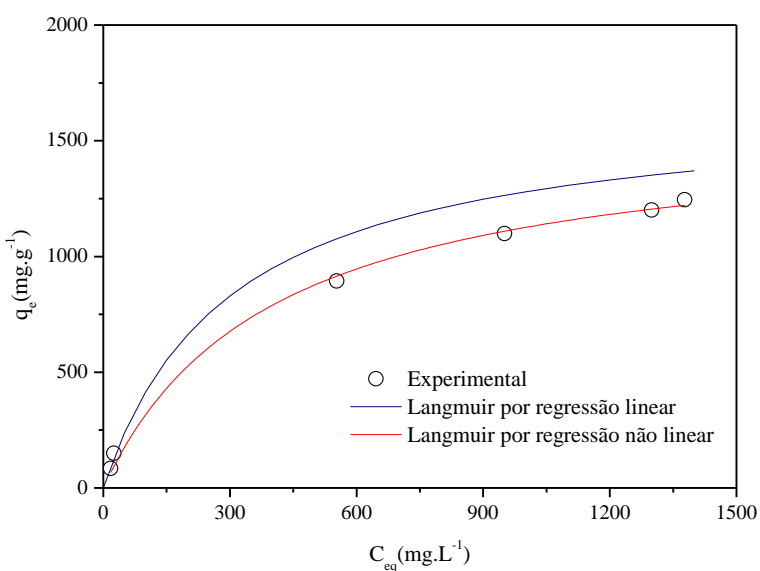

Figura 3 - Isotermas de Langmuir para a casca de pinhão in natura.

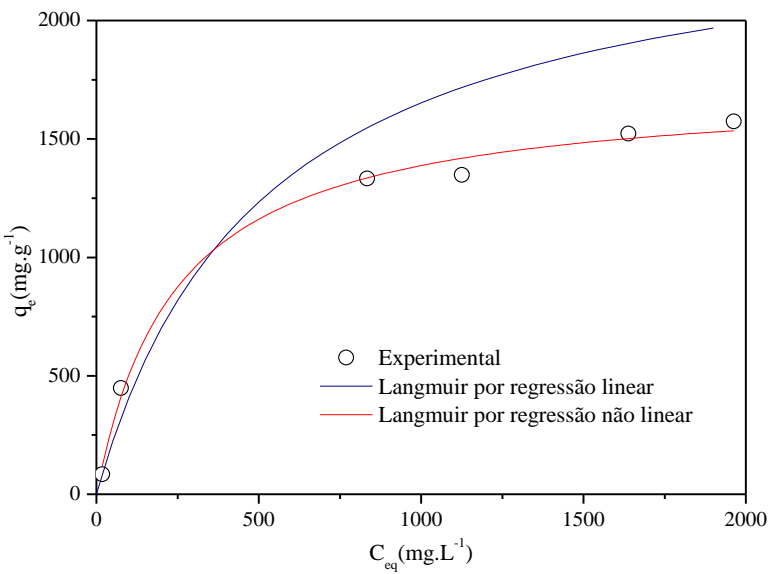

Figura 4 - Isotermas de Langmuir para a casca de pinhão tratada com $\mathrm{HCl}$.

$$
q_{e}=\frac{q_{m} K_{L} C_{e q}}{1+K_{L} C_{e q}} \text { ou linearizada: } \frac{1}{q_{e}}=\frac{1}{q_{m}}+\frac{1}{q_{m} K_{L} C_{e q}}
$$

onde $\mathrm{q}_{\mathrm{m}}\left(\mathrm{mg} \cdot \mathrm{g}^{-1}\right)$ é a capacidade máxima de adsorção e $\mathrm{K}_{\mathrm{L}}\left(\mathrm{L} \cdot \mathrm{mg}^{-1}\right)$ é a constante de Langmuir, relacionada com a energia de ligação. 
Tabela 1 - Parâmetros para a isoterma de Langmuir

\begin{tabular}{l|ccc|ccc}
\hline \multirow{2}{*}{$\begin{array}{l}\text { Metodologia para } \\
\text { estimação dos parâmetros }\end{array}$} & \multicolumn{3}{|c|}{ Casca de Pinhão in natura } & \multicolumn{3}{c}{ Casca de Pinhão tratada com HCl } \\
\cline { 2 - 7 } Regressão linear & $\mathrm{K}_{\mathrm{L}}\left(\mathrm{L} \cdot \mathrm{mg}^{-1}\right)$ & $\mathrm{q}_{\mathrm{m}}\left(\mathrm{mg} \cdot \mathrm{g}^{-1}\right)$ & $\mathrm{R}^{2}$ & $\mathrm{~K}_{\mathrm{L}}\left(\mathrm{L} \cdot \mathrm{mg}^{-1}\right)$ & $\mathrm{q}_{\mathrm{m}}\left(\mathrm{mg}_{\mathrm{g}} \mathrm{g}^{-1}\right)$ & $\mathrm{R}^{2}$ \\
\cline { 2 - 8 } Regressão não linear & 0,003302 & 1666,67 & 0,9916 & 0,001948 & 2500,00 & 0,9787 \\
Ryyyyyyy & 0,002510 & 1573,84 & 0,9958 & 0,004120 & 1724,17 & 0,9939 \\
\hline
\end{tabular}

As Figuras 5 e 6 apresentam os resultados experimentais e isoterma de adsorção de Freundlich (Equação 3), obtida para o pH=12 e temperatura de $298 \mathrm{~K}$, para a casca de pinhão in natura e tratada com ácido clorídrico, respectivamente. Os parâmetros da isoterma de Freundlich (Tabela 2) foram determinados por regressão linear (Mínimos Quadrados) e regressão não linear (método Simplex).

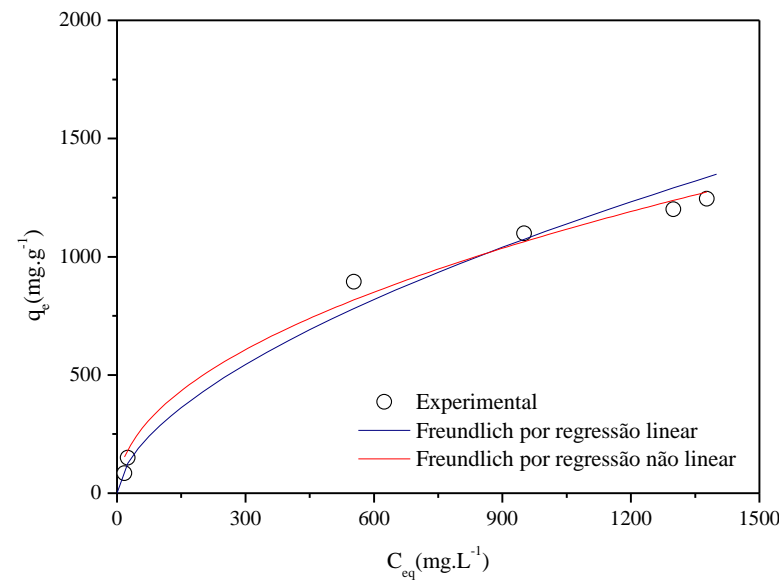

Figura 5 - Isotermas de Freundlich para a casca de pinhão in natura.

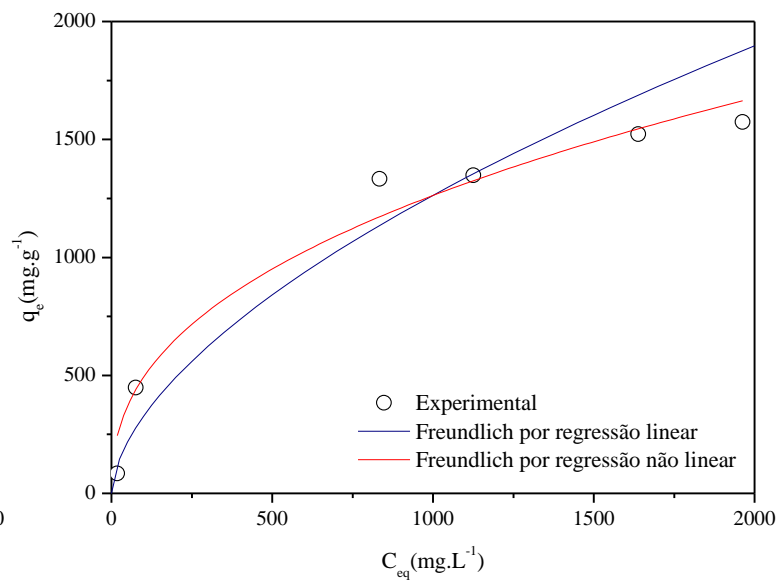

Figura 6 - Isotermas de Freundlich para a casca de pinhão tratada com $\mathrm{HCl}$.

$q_{e}=K_{F} C_{e q}^{1 / n}$ ou linearizada: $\ln \left(q_{e}\right)=\ln \left(K_{F}\right)+\frac{1}{n} \ln \left(C_{e q}\right)$

onde $K_{F}\left(\mathrm{~L}_{\mathrm{g}} \mathrm{g}^{-1}\right)$ é o coeficiente de adsorção de Freundlich e $n$ é um parâmetro adimensional.

Tabela 2 - Parâmetros para a isoterma de Freundlich

\begin{tabular}{|c|c|c|c|c|c|c|}
\hline \multirow{2}{*}{$\begin{array}{c}\text { Metodologia para } \\
\text { estimação dos parâmetros }\end{array}$} & \multicolumn{3}{|c|}{ Casca de Pinhão in natura } & \multicolumn{3}{|c|}{ Casca de Pinhão tratada com $\mathrm{HCl}$} \\
\hline & $\mathrm{K}_{\mathrm{F}}\left(\mathrm{L} \cdot \mathrm{g}^{-1}\right)$ & $\mathrm{n}$ & $\mathrm{R}^{2}$ & $\mathrm{~K}_{\mathrm{F}}\left(\mathrm{L} \cdot \mathrm{g}^{-1}\right)$ & $\mathrm{n}$ & $\mathrm{R}^{2}$ \\
\hline Regressão linear & 18,91 & 1,697 & 0,9867 & 21,92 & 1,703 & 0,9378 \\
\hline Regressão não linear & 37,85 & 2,055 & 0,9866 & 74,89 & 2,445 & 0,9613 \\
\hline
\end{tabular}

Ao observar os resultados apresentados pelas Figuras 3 a 6 percebe-se que o modelo de Langmuir ajustou-se melhor aos dados experimentais sugerindo, portanto, que não existe qualquer interação entre as moléculas de adsorbado e que a adsorção ocorre em monocamada, tanto para a casca in natura quanto para a casca tratada com $\mathrm{HCl}$. $\mathrm{Na}$ análise do modelo de Freundlich, em relação ao valor de $n$ obtido, considerando que foi maior que 1 (um) para as situações avaliadas, pode-se dizer que a distribuição dos sítios energéticos tende a variar com a densidade de adsorção.

Com relação aos métodos para a estimação dos parâmetros, observa-se que, para todas as situações avaliadas (Tabelas 1 e 2), o método de regressão não linear apresentou melhores resultados, ou seja, apresentou maior coeficiente de determinação para os ajustes realizados. 
Isso pode ser explicado pelo fato de que quando a equação é linearizada se tem uma transformação da equação de não linear para linear, que gera uma distorção do erro experimental, além das hipóteses assumidas pelo método de mínimos quadrados linearizado, de que dados experimentais possuem comportamento linear, que não é o caso do processo de adsorção (Armagan e Toprak, 2013; Osmari et al., 2013).

Em relação à capacidade de adsorção, pelo coeficiente $q_{m}$ do modelo de Langmuir, conforme mostra a Tabela 1, pode-se dizer que a casca de pinhão tratada com $\mathrm{HCl}$ obteve melhor desempenho. Esta observação é mantida quando se comparam os valores do parâmetro $K_{F}$ da isoterma de Freundlich (Tabela 2), que também sugere que a casca de pinhão tratada com $\mathrm{HCl}$ obteve melhor desempenho, apesar desta vantagem não ser muito significativa.

Por fim, também foram realizados ensaios experimentais para a determinação da cinética de adsorção. Os ensaios foram realizados para as condições de: $\mathrm{pH}=12, \mathrm{~V}=30 \mathrm{~mL}$, $\mathrm{m}_{\text {adsorvente }}=0,015 \mathrm{~g}$ e $\mathrm{C}_{0}=30 \mathrm{mg} \cdot \mathrm{L}^{-1}$. Foram avaliados os modelos de pseudoprimeira ordem (Equação 4), pseudossegunda ordem (Equação 5) e modelo de difusão intrapartícula proposto por Weber e Morris (Equação 6) (Spinelli et al., 2005).

$$
\begin{aligned}
& q=q_{e}\left(1-e^{k_{1} t}\right) \\
& q=\frac{k_{2} q_{e}^{2} t}{1+k_{2} q_{e} t} \\
& q=f t^{1 / 2}
\end{aligned}
$$

onde $\mathrm{k}_{1}\left(\mathrm{~min}^{-1}\right)$ é a constante de velocidade de pseudoprimeira ordem, $\mathrm{k}_{2}\left(\mathrm{~g} \cdot \mathrm{mg}^{-1} \cdot \mathrm{min}^{-1}\right)$ é a constante de velocidade de pseudossegunda ordem e $f\left(\mathrm{mg}_{\mathrm{g}} \mathrm{g}^{-1} \cdot \mathrm{min}^{-1 / 2}\right)$ é a constante de transporte intrapartícula.

As Tabelas 3 e 4 apresentam os coeficientes de determinação obtidos para os ajustes dos modelos propostos, para a casca de pinhão in natura e tratada com $\mathrm{HCl}$, respectivamente.

Os resultados apresentados nas Tabelas 3 e 4 confirmam que o modelo de pseudossegunda ordem representa melhor o ajuste dos dados experimentais, tanto para a casca de pinhão in natura quanto para a tratada com ácido clorídrico. Sendo assim, sugere-se que a adsorção é controlada pela difusão externa.

Tabela 3 - Coeficientes de determinação $\left(\mathrm{R}^{2}\right)$ para o ajuste dos modelos propostos para a casca de pinhão in natura

\begin{tabular}{c|c|c|c}
\hline T $\left({ }^{\circ} \mathrm{C}\right)$ & Pseudoprimeira ordem & Pseudossegunda ordem & Difusão intrapartícula \\
\hline 25 & 0,9880 & 0,9933 & 0,9259 \\
30 & 0,9919 & 0,9905 & 0,9726 \\
35 & 0,9468 & 0,9934 & 0,8756 \\
40 & 0,8743 & 0,9930 & 0,8870 \\
\hline
\end{tabular}


Tabela 4 - Coeficientes de determinação $\left(\mathrm{R}^{2}\right)$ para o ajuste dos modelos propostos para a casca de pinhão tratada com $\mathrm{HCl}$

\begin{tabular}{c|c|c|c}
\hline $\mathrm{T}\left({ }^{\circ} \mathrm{C}\right)$ & Pseudoprimeira ordem & Pseudossegunda ordem & Difusão intrapartícula \\
\hline $25^{\circ} \mathrm{C}$ & 0,9225 & 0,9941 & 0,8484 \\
$30^{\circ} \mathrm{C}$ & 0,9474 & 0,9894 & 0,9626 \\
$35^{\circ} \mathrm{C}$ & 0,9834 & 0,9960 & 0,8730 \\
$40^{\circ} \mathrm{C}$ & 0,9806 & 0,9967 & 0,8599 \\
\hline
\end{tabular}

$\mathrm{Na}$ Tabela 5 são apresentados os valores da constante cinética de pseudossegunda ordem, modelo que obteve melhor ajuste, para a casca de pinhão in natura e tratada com ácido clorídrico. Pode-se observar que a constante cinética é maior para a casca de pinhão que obteve tratamento com $\mathrm{HCl}$ em todas as temperaturas avaliadas. Isso acontece pelo fato da difusão intrapartícula limitar mais o processo na casca in natura que no processo da casca com o tratamento ácido, pois na casca tratada o poro está mais disponível. Assim, como o tempo para atingir o equilíbrio é maior na difusão interna do que na externa, este limita a adsorção no adsorvente in natura.

Tabela 5 - Constante cinética do modelo de pseudossegunda ordem $\left(\mathrm{k}_{2} \mathrm{em} \mathrm{g} \cdot \mathrm{mg}^{-1} \cdot \mathrm{min}^{-1}\right)$

\begin{tabular}{c|cccc}
\hline $\mathrm{T}\left({ }^{\circ} \mathrm{C}\right)$ & 25 & 30 & 35 & 40 \\
\hline Casca de pinhão In natura & 0,019302 & 0,014593 & 0,024185 & 0,023047 \\
Casca de pinhão tratada com HCl & 0,033383 & 0,014741 & 0,033108 & 0,035018 \\
\hline
\end{tabular}

\section{CONCLUSÕES}

Este estudo demonstrou que o tratamento ácido da casca de pinhão influenciou a cinética de adsorção e a quantidade de corante adsorvida. O modelo de pseudossegunda ordem foi o que melhor se ajustou aos resultados experimentais e se obteve maior constante cinética para a casca de pinhão tratada com ácido clorídrico. A velocidade de adsorção foi modificada pelo efeito da difusão interna, a qual limita mais o processo da casca in natura do que na casca tratada, tornando assim o processo de adsorção mais rápido nesta.

A capacidade de adsorção da casca de pinhão foi levemente influenciada pelo tratamento com $\mathrm{HCl}$, obtendo-se melhores resultados para a casca tratada. $\mathrm{O}$ modelo de Langmuir ajustou-se melhor aos resultados experimentais, sugerindo que a adsorção ocorrem em monocamada e se tenha sítios homogêneos.

\section{REFERENCIAS}

AKSU, Z. Application of biosorption for the removal of organic pollutants: a review. Process Biochemistry, v. 40, p. 997-1026, 2005.

ARMAGAN, B.; TOPRAK, F. Optimum isotherm parameters for reactive azo dye onto pistachio nut shells: Comparison of linear and non-linear methods. Pol. J. Environ. Stud., v. 22, n. 4, p. 1007-1011, 2013.

BIELSKA, M.; PROCHASKA, K. Dyes separation by means of cross-flow ultrafiltration of micellar solutions. Dyes and Pigments, v. 74, p. 410-415, 2007. 
FOLETTO, E. L.; JAHN, S. L.; MOREIRA, R.F.P.M. Hydrothermal preparation of Zn2SnO4 nanocrystals and photocatalytic activity in the degradation of leather dye, J. Appl. Electrochem., v. 40, p. 59-63, 2010.

KARADAG, D.; TOK, S.; AKGUL, E.; ULUCAN, K.; EVDEN, H.; KAYA, M. Combining adsorption and coagulation for the treatment of azo and anthraquinone dyes from aqueous solution, Ind. Eng. Chem. Res., v. 45, p. 3969-3973, 2006.

LIMA, C. E; ROYER, B.; VAGHETTI, C. P. J.; BRASIL, L. J., Adsorption of Cu(II) on Araucaria angustifolia wastes: Determination of optimal conditions by statistic design of experiments. Journal of Hazardous Materials, v. 140, p. 211-220, 2007.

MOHORCIC, M.; TEODOROVIC, S.; GOLOB, V.; FRIEDRICH, V. Fungal and enzymatic decolourisation of artificial textile dye baths, Chemosp., v. 63, p. 1709-1717, 2006.

OSMARI, T. A.; GALLON, R.; SCHWAAB, M.; BARBOSA-COUTINHO, E.; SEVERO JR, J. B.; PINTO, J. C.. Statistical Analysis of Linear and Non-linear Regression for the Estimation of Adsorption Isotherm Parameters. Adsorption Science \& Technology, v. 31, p. 433-458, 2013.

PAPIC, S.; KOPRIVANACA, N.; BOZIC, A.L. Removal of reactive dyes from wastewater using Fe (III) coagulants, J. Soc. Dyers Color, v. 116, p. 352-358, 2000.

RAFATULLAH, M., SULAIMAN, O., HASHIM, R., AHMAD, A.. Adsorption of methylene blue on low-cost adsorbents: A review. J Hazard Mater, v. 177, p. 70-80, 2010.

REIFE, A.; FREMANN, H.S. Environmental Chemistry of Dyes and Pigment, New York: John Wiley Inc., 1996.

SPINELLI, V. A.; LARANJEIRA, M. C. M.; FAVERE, V. T.; KIMURA, I. Y. Cinética e equilíbrio de adsorção dos oxiânions Cr (VI), Mo (VI) e Se (VI) pelo sal de amônio quaternário de quitosana. Polímeros. v. 15, n. 3, p. 218-223, 2005. 